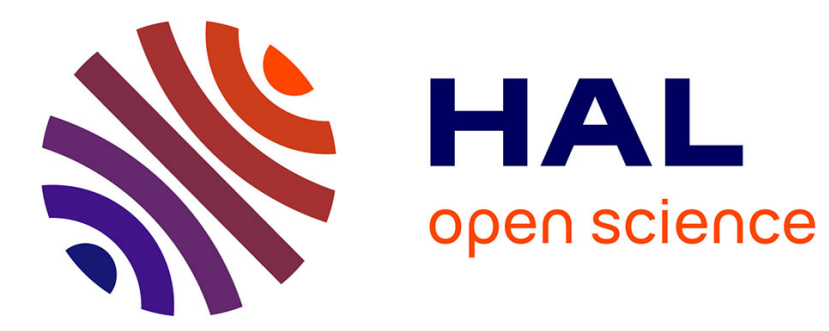

\title{
Wavelength Stabilization of Extended-Cavity Tapered Lasers with Volume Bragg Gratings
}

Gaëlle Lucas-Leclin, Patrick Georges, Jürgen Weber, Mark T. Kelemen, Bernd Sumpf, Götz Ebert

\section{- To cite this version:}

Gaëlle Lucas-Leclin, Patrick Georges, Jürgen Weber, Mark T. Kelemen, Bernd Sumpf, et al.. Wavelength Stabilization of Extended-Cavity Tapered Lasers with Volume Bragg Gratings. Conference on Laser and Electro-Optics (CLEO), May 2006, Baltimore, United States. hal-00128177

\section{HAL Id: hal-00128177 \\ https://hal.science/hal-00128177}

Submitted on 31 Jan 2007

HAL is a multi-disciplinary open access archive for the deposit and dissemination of scientific research documents, whether they are published or not. The documents may come from teaching and research institutions in France or abroad, or from public or private research centers.
L'archive ouverte pluridisciplinaire HAL, est destinée au dépôt et à la diffusion de documents scientifiques de niveau recherche, publiés ou non, émanant des établissements d'enseignement et de recherche français ou étrangers, des laboratoires publics ou privés. 


\title{
Wavelength Stabilization of Extended-Cavity Tapered Lasers with Volume Bragg Gratings
}

\author{
G. Lucas-Leclin, P. Georges \\ Laboratoire Charles Fabry de l'Institut d'Optique, UMR 8501 \\ Centre Universitaire - Batiment 503, 91403 Orsay Cedex (France) \\ gaelle.lucas-leclin@iota.u-psud.fr
}

J. Weber, M. T. Kelemen

Fraunhofer Institut für Angewandte Festkörperphysik, Tullastr. 72, 79108 Freiburg, Germany

B. Sumpf, G. Erbert

Ferdinand-Braun-Institut für Höchtstfrequenztechnik, Albert-Einstein Strasse 11, 12489 Berlin, Germany

\begin{abstract}
We describe the strong wavelength-stabilization of high-brightness extended-cavity lasers based on 808-nm and 976-nm tapered amplifiers by use of volume Bragg gratings. Single-frequency operation has been obtained in a robust and simple design.

(C) 2006 Optical Society of America

OCIS codes : 140.5960 Semiconductor Lasers, 050.7330 Volume Holographic Gratings
\end{abstract}

\section{Introduction}

Narrow spectrum, high-brightness semiconductor lasers emitting in the near infrared are of great interest for a wide range of applications, from direct pumping of rare-earth solid-state lasers to nonlinear frequency conversion to the visible for medicine or spectroscopy. The most promising design proposed for high brightness emission in the 1-W power range is the tapered laser diode [1]. It consists in a single-mode ridge acting as a spatial filter and a large tapered amplifier section. Several realizations have been described in the literature, demonstrating output powers as high as $4 \mathrm{~W}$ in a nearly diffraction-limited beam [2-5]. However the large emission spectrum of these devices remains a strong limitation for demanding applications. A well-known, efficient way to control the laser spectrum is to use the tapered device as the gain medium in an extended-cavity with a spectrally selective component. Usually it consists of a diffraction grating in a Littrow configuration, which allows both tunability and spectral selectivity [6,7]

We proposed a new and simpler design for the numerous applications in which the emission wavelength is a priori known. The spectrally selective element is a volume reflection grating, which forces the laser emission to the Bragg wavelength whereas the output power and beam properties are imposed by the tapered amplifier structure.

\section{Description of the external cavity}

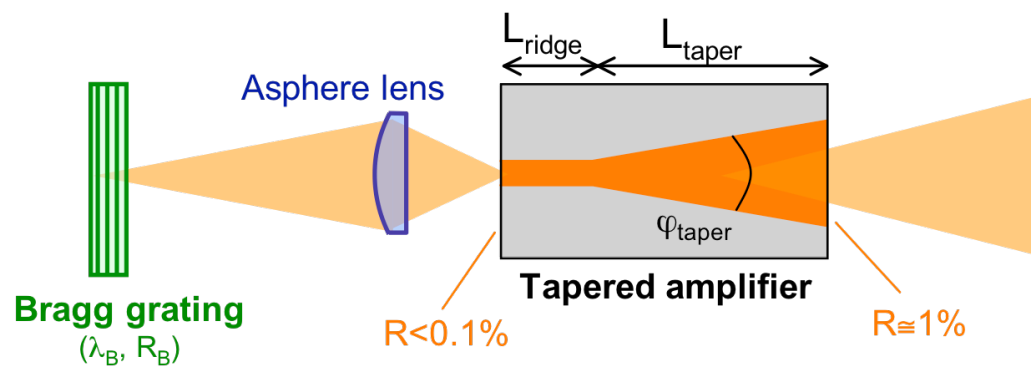

Figure 1 : Design of the extended-cavity tapered laser.

Our external cavity is very simple: on the first hand, the volume Bragg grating is realized in a photosensitive glass, which allows direct writing of periodic structures under UV illumination to make filters with the desired properties. Here we used commercial Bragg gratings, with reflectivity in the range $10 \%-40 \%$ and a reflection bandwidth of $0.5 \mathrm{~nm}$ or less [8]. On the second hand the laser medium is a tapered amplifier with an antireflection coating on the single-mode ridge side $(\mathrm{R}<0.1 \%)$, and a low reflection coating on the tapered side $(\mathrm{R} \sim 1 \%)$. On the ridge side the emission is focused into the Bragg grating with a high-NA asphere lens. Let's note that the focalization of the beam inside the grating results in a stronger control of the emitted wavelength and a lower sensitivity to cavity misalignments than in the case where the beam is collimated.

We have used this cavity scheme (Fig. 1) with different high-brightness tapered amplifiers: the first ones were single quantum well InGaAs/AlGaAs devices designed to operate at $980 \mathrm{~nm}$, with a 
filtering ridge section length of $500 \mu \mathrm{m}$, a taper section length of $2.5 \mathrm{~mm}$ and a taper angle of $6^{\circ}$ [4]. The second ones were based on the well-established GaAsP/AlGaAs layer structure designed at 808 $\mathrm{nm}$, with a $500 \mu \mathrm{m}$-long ridge and a $3.5 \mathrm{~mm}$-long $4^{\circ}$-angled tapered section [5]. For comparison tapered lasers with the same layer and transverse structure but a HR coating on the rear facet have been realized, demonstrating high beam quality for output powers up to $3 \mathrm{~W}$.

\section{Experimental characterization of the laser emission}

With both $976 \mathrm{~nm}$ and $808 \mathrm{~nm}$ amplifiers without optical feedback, no laser emission could be observed but only amplified spontaneous emission covering a large spectrum. In external cavity an output power of typically $1 \mathrm{~W}$ was emitted from the large tapered output for an operating current of about $4 \mathrm{~A}$. This output power was mainly limited by the performances of the semiconductor amplifiers, indeed we have measured similar characteristics with our volume gratings or with a broadband high reflectivity mirror. A strong laser emission at the Bragg wavelength was observed with our different gratings for any reflectivity in the range $10 \%$ to $40 \%$. As shown in fig. 2 , the sidemode suppression is $40 \mathrm{~dB}$, and the laser line is locked at the Bragg wavelength whatever the amplifier operating current or temperature, with a FWHM linewidth lower than 80 pm (OSAlimited). The single longitudinal mode operation of the $976.0 \mathrm{~nm}$ extended-cavity tapered laser was controlled with a scanning Fabry-Perot interferometer with a free spectral range of $8 \mathrm{GHz}$. A true single-frequency operation was obtained though some mode-hopping appeared if the operating conditions were changed. Very similar results have been demonstrated with the $808-\mathrm{nm}$ tapered sources and a Bragg grating characterized by $\lambda_{B}=807.3 \mathrm{~nm}$ and $R_{B}=40 \%$.
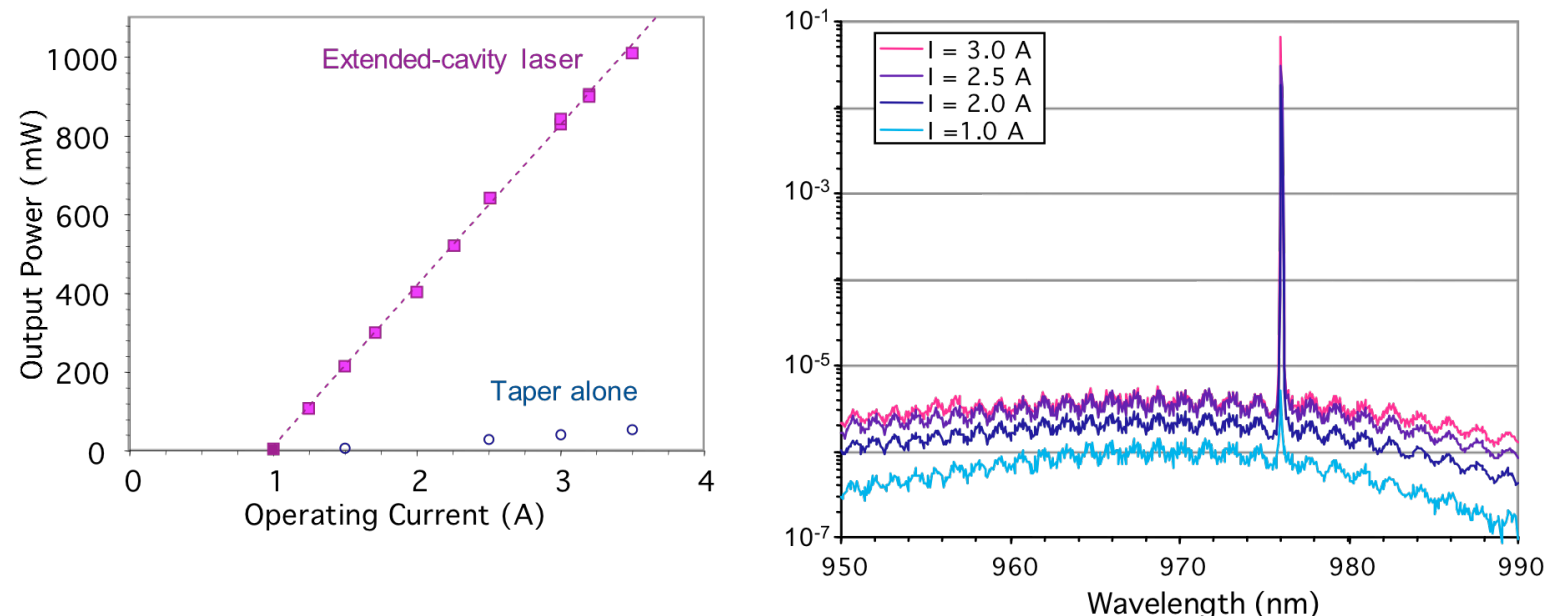

Figure 2 : (Right) Laser spectrum of the tapered source in extended-cavity, for different amplifier currents ; the Bragg wavelength is at $976.0 \mathrm{~nm}$, and the reflection coefficient of the grating at this wavelength is $10 \%$. (Left) Output powers for the tapered amplifier alone (ASE) and in extended-cavity laser operation $\left(\mathrm{T}=25^{\circ} \mathrm{C}\right)$.

\section{Conclusion}

We have demonstrated an output power of $1 \mathrm{~W}$ from high-brightness tapered amplifiers in a new compact and robust external cavity operating at the fixed wavelength imposed by a volume Bragg grating. Such laser sources are adapted for second harmonic generation in non-linear crystals towards the visible spectrum (488 nm, $405 \mathrm{~nm}$ e.g.), thanks to their narrow linewidth and good beam quality. We are now looking forward improving the output power of these sources with improved amplifiers.

\section{References}

[1] J.N. Walpole, "Semiconductor amplifiers and lasers with tapered gain regions", Opt. Quantum Electron. 28, 623-645 (1996)

[2] B. Sumpf et al, "High-brightness $735 \mathrm{~nm}$ tapered diode lasers", Elec. Lett. 38 (4), 183-184 (2002)

[3] M.T. Kelemen et al, "High power high-brightness ridge-waveguide tapered diode lasers at $940 \mathrm{~nm}$ ", in Proceedings of SPIE 4648, 75-81 (2002)

[4] M.T. Kelemen et al, "High-Power High-Brightness Tapered Diode Lasers and Amplifiers", in Proceedings of SPIE 5723 (2005)

[5] A. Knauer et al, "High-power 808 nm lasers with a super-large optical cavity", Semicond. Sci. Technol. 20, 621-624 (2005)

[6] D. Mahuys et al, "1W CW, diffraction-limited, tunale external-cavity semiconductor laser", Elec. Lett. 29 (14), $1254-1255$ (1993)

[7] A. Goyal et al., "Stable single-frequency operation of a high-power external-cavity tapered diode laser at 780 nm", APL 71 (10), 1296-1298 (1997).

[8] B. Volodin et al., "Wavelength stabilization and spectrum narrowing of high-power multimode laser diodes and arrays by use of volume Bragg gratings", Opt. Lett. 29 (16), 1891-1894 (2004)

\section{Aknowledgments}

The authors thank the European Community for financial support under the www.BRIGHT.eu program (IP 511722). 